\section{One-shot parallel complex Fourier-domain optical coherence tomography using a spatial carrier frequency}

\author{
Peng $\mathrm{Bu}^{\mathrm{a}, \mathrm{b}}$ Xiangzhao Wang, ${ }^{\mathrm{a}, \mathrm{b}}$ and Osami Sasaki ${ }^{\mathrm{c}}$ \\ ${ }^{a}$ Chinese Academy of Sciences, Information Optics \\ Laboratory, Shanghai Institute of Optics and Fine \\ Mechanics, Shanghai 201800, China \\ ${ }^{\mathrm{b}}$ Graduate School of the Chinese Academy of Sciences, \\ Beijing 100039, China \\ ${ }^{\mathrm{C}}$ Niigata University, Faculty of Engineering, Niigata-shi 950- \\ 2181, Japan \\ E-mail: wxz26267@siom.ac.cn
}

\begin{abstract}
We propose a novel method of one-shot parallel complex Fourier-domain optical coherence tomography using a spatial carrier frequency for full range imaging. The spatial carrier frequency is introduced into the 2-D spectral interferogram in the lateral direction by using a tilted reference wavefront. This spatial-carrier-contained 2-D spectral interferogram is recorded with one shot of a 2-D CCD camera, and is Fourier-transformed in the lateral direction to obtain a 2-D complex spectral interferogram by a spatial-carrier technique. A full-range tomogram is reconstructed from the 2-D complex spectral interferogram. The principle of this method is confirmed by cross-sectional imaging of a glass slip object. () 2008 Society of Photo-Optical Instrumentation Engineers. [DOI: 10.1117/1.2931575]
\end{abstract}

Subject terms: optical coherence tomography; interferometry; spatial carrier frequency.

Paper 080025LR received Jan. 13, 2008; revised manuscript received Mar. 27, 2008; accepted for publication Mar. 28, 2008; published online May 23, 2008.

Recently Fourier domain optical coherence tomography $(\text { FDOCT })^{1}$ has attracted great attention because of its high speed and sensitivity compared to the conventional timedomain OCT (TDOCT). However, FDOCT suffers from the complex conjugate ambiguity due to its straightforward Fourier transformation of the real-valued spectral interferogram, which reduces the available imaging depth range to half the original area. In addition, the presence of dc and autocorrelation noises obscures the FDOCT image.

Over the last few years, various methods to reconstruct a complex spectral interferogram in FDOCT for full-range imaging have been proposed. First a phase-shifting method was used, ${ }^{2}$ where multiple phase-shifted spectral interferograms are detected to reconstruct a complex spectral interferogram. However, it requires high stability of the interferometer and the sample, which makes it difficult to apply to in vivo measurement. Simultaneously, phase-shifting methods were proposed to avoid this problem, but these methods increase the system complexity and cost because of the use of $3 \times 3$ fiber couplers ${ }^{3}$ or polarization-based optical components.

0091-3286/2008/\$25.00 @ 2008 SPIE
Recently, carrier-frequency-based methods have been employed. In one method a carrier frequency is introduced into the spectral interferogram corresponding to one lateral measurement position, ${ }^{5,6}$ i.e., an A scan. In another, a carrier frequency is introduced into successive A scans corresponding to different lateral measurement positions, i.e., a B scan. ${ }^{\text {T-9 }}$

The common characteristic of all the preceding methods is that the carrier frequency is generated in the form of time-dependent phase modulation with various kinds of frequency shifters or phase modulators. When a time-varying object is measured with these methods (e.g., in vivo imaging), a high temporal carrier frequency is required. For time-varying objects, a spatial carrier technique is more suitable, because its carrier frequency is generated by spatially dependent phase modulation at an instant of time. ${ }^{10}$

In this letter, we combine a spatial carrier technique with parallel FDOCT ${ }^{11}$ to achieve a one-shot parallel complex FDOCT for full-range imaging, in which a spatial carrier frequency is introduced into the two-dimensional (2-D) spectral interferogram along the lateral positions by using a tilted reference wavefront. This spatial-carrier-contained 2-D spectral interferogram is recorded in a single shot at an instant of time with a 2-D CCD camera. A similar optical scheme of reference wavefront tilt has been previously used to realize a one-shot phase-shifting FDOCT, ${ }^{12}$ where five spectral fringes with different phase offsets are captured by a 2-D CCD camera to obtain a 1-D complex spectral interferogram for one lateral position with a five-frame phaseshifting algorithm. Here, we use the reference wavefront tilt to reconstruct the 2-D complex spectral interferogram by Fourier transformation ${ }^{13}$ from the spatial-carrier-contained 2-D spectral interferogram recorded with a single shot of the 2-D CCD camera

A schematic diagram of the proposed one-shot parallel complex FDOCT using a spatial carrier frequency is shown in Fig. 1. The optical setup is based on a parallel FDOCT using linear illumination and 1-D imaging optics. ${ }^{11}$ The light from a broadband source is collimated by a lens (L1) and split into the reference and probing beams by a cube beamsplitter (BS). A linear illumination beam generated by a cylindrical lens (CL) is focused through two lenses (L2 and L3) onto the reference mirror (RM) and a sample along the vertical direction ( $x$ axis), respectively. The two linear

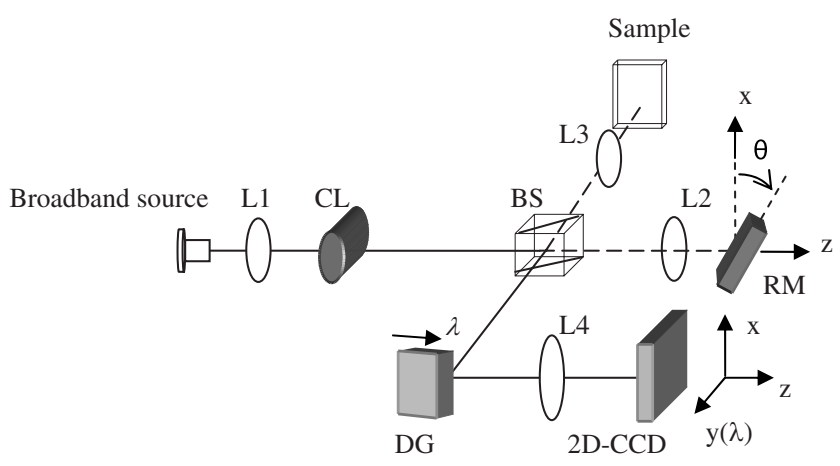

Fig. 1 Schematic diagram of one-shot parallel complex Fourierdomain optical coherence tomography using a spatial carrier frequency. CL, cylindrical lens; $B S$, cube beamsplitter; $L 1, \ldots, L 4$, lenses; RM, reference mirror; DG, diffraction grating; $\lambda$ is the wavelength of the light; $\theta$ is the tilt angle of reference mirror. 
beams reflected back from the reference mirror and from within the sample provide a 2-D spectral interferogram with a spectrometer consisting of a diffraction grating (DG), an achromatic lens (L4), and a 2-D CCD camera. The wavelength is dispersed on the CCD along the horizontal direction ( $y$ axis). The line-field-illuminated lateral points on the sample are imaged on the camera along the $x$-axis direction. Thus, the two dimensions of the 2-D spectral interferogram are in the spectral direction ( $y$ axis) and the lateral direction ( $x$ axis), respectively. By tilting the reference mirror along the $x$-axis direction as shown in Fig. 1 , resulting in a tilted reference wavefront, a spatial carrier frequency is introduced into the 2-D spectral interferogram in the lateral direction, which can be expressed as

$g(k, x)=g_{0}(k, x)+2 S(k) \sum_{n}\left(\alpha_{n} \beta_{0}\right)^{1 / 2} \cos \left[2 k\left(z_{n}+x \sin \theta\right)\right]$,

where $k=2 \pi / \lambda$ is the wave number; $z_{n}$ is the one-way optical path difference between the beam reflected by the $n$ 'th interface within the sample and the beam reflected by the reference mirror; $x$ is the lateral position; $\theta$ is the tilt angle of the reference mirror along the $x$-axis direction; $g_{0}(k, x)$ includes the dc and autocorrelation terms; $\alpha_{n}$ is the reflectivity of the $n$ 'th interface of the sample; $\beta_{0}$ is the reflectivity of the reference mirror; and $S(k)$ is the spectrum of the light source.

Equation (1) can be rewritten as

$$
\begin{aligned}
g(k, x)= & g_{0}(k, x)+\sum_{n} b_{n}(k) \exp \left(i 2 \pi f_{x 0} x\right) \\
& +\sum_{n} b_{n}^{*}(k) \exp \left(-i 2 \pi f_{x 0} x\right),
\end{aligned}
$$

where $\phi_{n}(k)=2 k z_{n}, \quad b_{n}(k)=S(k)\left(\alpha_{n} \beta_{0}\right)^{1 / 2} \exp \left[i \phi_{n}(k)\right], \quad f_{x 0}$ $=(2 \sin \theta) / \lambda$, and ${ }^{*}$ denotes the complex conjugate operator. The Fourier transform of $g(k, x)$ with respect to lateral position $x$ for each $k$ is given by

$$
G\left(k, f_{x}\right)=G_{0}\left(k, f_{x}\right)+\sum_{n} B_{n}\left(k, f_{x}-f_{x 0}\right)+\sum_{n} B_{n}^{*}\left(k, f_{x}+f_{x 0}\right),
$$

where $G$ and $B$ are the Fourier spectra of $g$ and $b$, respectively, $f_{x}$ is the spatial frequency in the lateral direction, and $f_{x 0}$ is the lateral spatial carrier frequency caused by the tilted wavefront of the reference mirror. When the lateral spatial variations of $g_{0}, b_{n}$, and $\phi_{n}$ are slow compared with the spatial carrier frequency $f_{x 0}$, the Fourier spectra in Eq. (3) are separated from each other. By frequency-domain bandpass filtering, we can obtain the spectrum of $\Sigma_{n} B_{n}\left(k, f_{x}-f_{x 0}\right)$, and translate it by $f_{x 0}$ toward the origin to obtain $\Sigma_{n} B_{n}\left(k, f_{x}\right)$. Then taking the inverse Fourier transform of $\sum_{n} B_{n}\left(k, f_{x}\right)$ with respect to $f_{x}$ for each $k$ gives the complex spectral interferogram as follows:

$g_{\text {comp }}(x, k)=\sum_{n} b_{n}(k)=\sum_{n} S(k)\left(\alpha_{n} \beta_{0}\right)^{1 / 2} \exp \left[i \phi_{n}(k)\right]$.

The inverse Fourier transform of $g_{\text {comp }}(x, k)$ with respect to $k$ for each $x$ yields
$I(x, z)=\sum_{n}\left(\alpha_{n} \beta_{0}\right)^{1 / 2} \Gamma\left(z-2 z_{n}\right)$,

where $\Gamma$ is the first-order electric field correlation function. From the amplitude of $I(x, z)$ a tomogram free of the complex conjugate ambiguity as well as dc and autocorrelation noises is obtained, leading to a doubled imaging depth range. As a result, full-range complex parallel FDOCT imaging is achieved with a single shot of the 2-D CCD camera.

Cross-sectional imaging of a glass slip object was performed to confirm the principle of our method. The system shown in Fig. 1 was built. The broadband light source used in the experiment was a superluminescent diode (SLD) with a central wavelength of $798 \mathrm{~nm}$ and a 19-nm full width at half maximum (FWHM) that provides a depth resolution of $15 \mu \mathrm{m}$ in air. The spectrometer consisted of a diffraction grating (600 grooves/mm), an achromatic lens with a focal length of $250 \mathrm{~mm}$, and a 2-D CCD camera with a frame rate of $60 \mathrm{~Hz}$ (9 bits, $648 \times 494$ pixels, 7.4 $\times 7.4 \mu \mathrm{m}$ pixel size). The spectrometer has a designed spectral resolution of $0.05 \mathrm{~nm}$, allowing for a full-range system imaging depth of $6.4 \mathrm{~mm}$ in air.

The optical system for linear imaging in the lateral direction has a magnification of 2.5 , an imaging length of $1.1 \mathrm{~mm}$, and a lateral resolution of $6 \mu \mathrm{m}$ determined by the pixel size of the CCD camera. However, the lateral resolution will be deteriorated in practice by the coherent crosstalk in the lateral direction when measuring biological samples, which can be suppressed by using a spatially incoherent light source such as a thermal light source, at the price of having only a small available optical power. ${ }^{11}$ The lateral imaging length and resolution respectively determine the spatial frequency resolution and maximum available spatial frequency in the lateral direction, according to the sampling theorem.

By tilting the reference mirror at an appropriate angle $\theta$ of $0.65 \mathrm{deg}$, we introduced a spatial carrier frequency $f_{x 0}$ of $28 \mathrm{~mm}^{-1}$ at the central wavelength of $798 \mathrm{~nm}$ into the 2-D spectral interferogram in the lateral direction, which separated the interferogram's lateral spectra from each other. It should be noted that in order to achieve the best separation of the lateral spectrum, and thus the best suppression of the complex conjugate ambiguity, the tilt angle of the reference mirror is preferably chosen such that the introduced spatial carrier frequency is equal to half the maximum available spatial frequency determined by the lateral imaging resolution. In this case the lateral spectrum of the measured object will be shifted to the center of the half Fourier space, where the maximum bandwidth of the lateral spectrum that can be separated is obtained.

Figure 2(a) shows the 2-D spectral interferogram with spatial carrier in the lateral direction recorded by a single shot of the 2-D CCD camera. Its Fourier spectrum with respect to the lateral position was obtained by fast Fourier transformation along the lateral position $x$, as shown in Fig. 2(b). In Fig. 2(b) we can see that three spectra are separated from each other, centering at spatial frequencies of $f_{x}$ $= \pm f_{x 0}$ and $f_{x}=0$. The spectrum around the spatial carrier frequency $f_{x 0}$ was filtered out for each wavelength $\lambda$, and translated to the origin. Then the translated spectrum was inverse-Fourier-transformed along $f_{x}$ to obtain the 2-D complex spectral interferogram, from which a full-range 


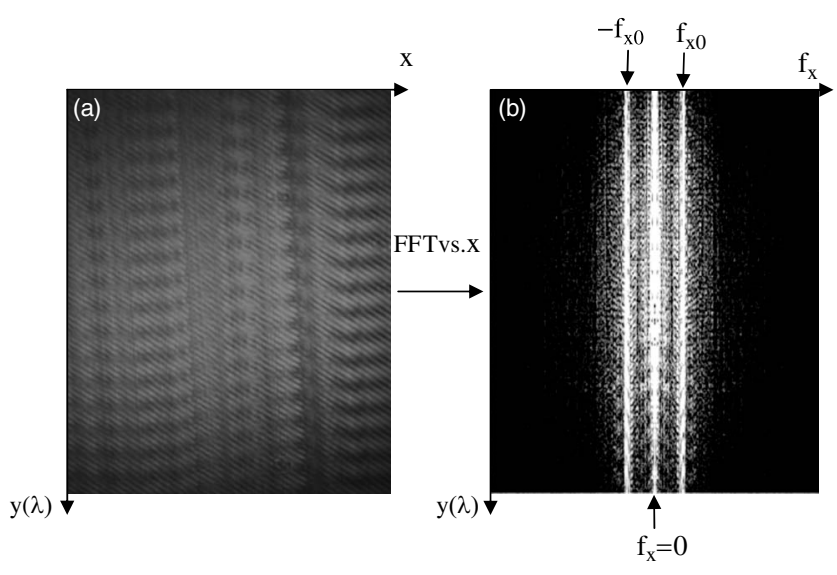

Fig. 2 (a) 2-D spectral interferogram with spatial carrier in lateral direction, and (b) its Fourier spectrum with respect to lateral position.

tomogram could be obtained. The processing time for calculating the full-range tomogram comprising 390 A-scan lines (640 pixels per A-scan line) is $\approx 30$ s with the algorithm implemented in MATLAB on a Pentium 4 CPU operating at $1.7 \mathrm{GHz}$.

Figure 3(a) shows the tomogram of the glass slip obtained by our proposed one-shot parallel complex FDOCT, where the complex conjugate ambiguity (CC) and the dc and autocorrelation (AC) noises are eliminated, compared with the tomogram obtained by a straightforward FDOCT as shown in Fig. 3(b). The suppression ratio of the complex conjugate ambiguity is better than $30 \mathrm{~dB}$. There are still some residual lines in the tomogram of Fig. 3(a), which are caused by the sidelobe generated by the Fourier transform of the filtering window function. These residual lines can be eliminated by utilizing a sidelobe-suppressed filtering window function such as Hamming window function. With a power at the sample of $\sim 1 \mathrm{~mW}$, the system sensitivity was measured to be $75 \mathrm{~dB}$, and a sensitivity falloff of $30 \mathrm{~dB}$ was observed at a distance of $3 \mathrm{~mm}$ from the zerodelay position.

In conclusion, a one-shot parallel complex FDOCT method using a spatial carrier frequency has been proposed
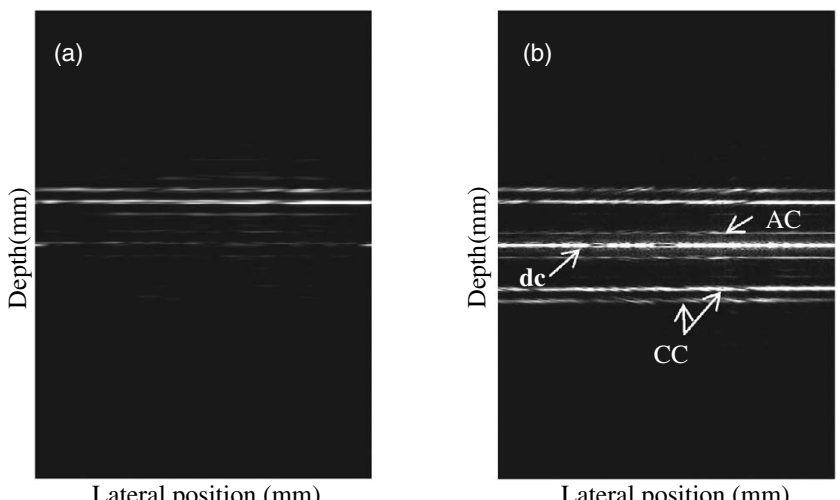

Fig. 3 Tomograms of glass slip object obtained by (a) one-shot parallel complex FDOCT using spatial carrier frequency and (b) straightforward FDOCT. Each tomogram corresponds to a crosssection area of $6.4 \mathrm{~mm}$ (depth) $\times 1.1 \mathrm{~mm}$ (lateral distance). for full-range imaging. A reference beam with tilted wavefront is used to introduce the spatial carrier frequency into the 2-D spectral interferogram. A full-range FDOCT tomogram is retrieved from a single spatial-carrier-contained 2-D spectral interferogram by a Fourier transform method. The advantages of the proposed method over the temporalcarrier-frequency method are that no additional phase modulator is required and the fringe washout effect due to the time-varying phase modulation is avoided.

It should be mentioned that our proposed system is still limited by the coherent crosstalk among the lateral points in the measurement of biological samples, because of the use of a line-field parallel FDOCT system. Recently the application of line-field parallel FDOCT to in vivo human retinal imaging has been demonstrated, ${ }^{14}$ where the integration time of the CCD camera is optimized to suppress the sensitivity degradation due to the sample's axial motion. We believe the proposed method here is helpful in achieving a fast and simple full-range parallel complex FDOCT system for in vivo imaging of thick biological tissue such as the anterior eye.

\section{Acknowledgments}

This work was supported by the National Natural Science Foundation of China under grant 60578051 and the Key Basic Research Program of Science and Technology Commission of Shanghai Municipality under grant 07JC14056.

\section{References}

1. R. A. Leitgeb, C. K. Hitzenberger, and A. F. Fercher, Performance of Fourier domain vs. time domain optical coherence tomography, Opt. Express 11, 889-894 (2003).

2. M. Wojtkowski, A. Kowalczyk, R. Leitgeb, and A. F. Fercher, Full range complex spectral optical coherence tomography technique in eye imaging, Opt. Lett. 27, 1415-1417 (2002).

3. M. Sarunic, M. A. Choma, C. Yang, and J. A. Izatt, Instantaneous complex conjugate resolved spectral domain and swept-source OCT using $3 \times 3$ fiber couplers, Opt. Express 13, 957-967 (2005).

4. B. J. Vakoc, S. H. Yun, G. J. Tearney, and B. E. Bouma, Elimination of depth degeneracy in optical frequency-domain imaging through polarization-based optical demodulation, Opt. Lett. 31, 362-364 (2006).

5. A. H. Bachmann, R. A. Leitgeb, and T. Lasser, Heterodyne Fourier domain optical coherence tomography for full range probing with high axial resolution, Opt. Express 14, 1487-1496 (2006).

6. J. Zhang, J. S. Nelson, and Z. Chen, Removal of a mirror image and enhancement of the signal-to-noise ratio in Fourier-domain optical coherence tomography using an electro-optic phase modulator, Opt. Lett. 30, 147-149 (2005).

7. Y. Yasuno, S. Makita, T. Endo, G. Aoki, M. Itoh, and T. Yatagai, Simultaneous B-M-mode scanning method for real-time full-range Fourier domain optical coherence tomography, Appl. Opt. 45, 18611865 (2006)

8. R. K. Wang, "In vivo full range complex Fourier domain optical coherence tomography, Appl. Phys. Lett. 90, 054103 (2007).

9. R. A. Leitgeb, R. Michaely, T. Lasser, and S. C. Sekhar, Complex ambiguity-free Fourier domain optical coherence tomography through transverse scanning, Opt. Lett. 32, 3453-3455 (2007).

10. M. Takeda and M. Kitoh, Spatiotemporal frequency multiplex heterodyne interferometry, J. Opt. Soc. Am. A 9, 1607-1614 (1992).

11. B. Grajciar, M. Pircher, A. F. Fercher, and R. A. Leitgeb, Parallel Fourier domain optical coherence tomography for in vivo measurement of the human eye, Opt. Express 13, 1131-1137 (2005).

12. Y. Yasuno, S. Makita, T. Endo, G. Aoki, H. Sumimura, M. Itoh, and T. Yatagai, One-shot-phase-shifting Fourier domain optical coherence tomography by reference wavefront tilting, Opt. Express 12, 6184$6191(2004)$

13. M. Takeda, H. Ina, and S. Kobayashi, Fourier-transform method of fringe-pattern analysis computer-based topography and interferometry, J. Opt. Soc. Am. 72, 156-160 (1982).

14. Y. Nakamura, S. Makita, M. Yamanari, M. Itoh, T. Yatagai, and Y. Yasuno, High-speed three-dimensional human retinal imaging by line-field spectral domain optical coherence tomography, Opt. Express 15, 7103-7116 (2007). 